\title{
Altruistic or Egocentric, or even Hypocritic? - A Cross-Cultural Study of the Safety Priority Choice of Autonomous Car
}

This paper was downloaded from TechRxiv (https://www.techrxiv.org).

\section{LICENSE}

CC BY 4.0

\section{SUBMISSION DATE / POSTED DATE}

27-09-2021 / 28-12-2021

\section{CITATION}

Mizumoto, Masaharu; Shigeharu, Ono,; Yumi, Okazaki,; Keigo, Kanetsuna, (2021): Altruistic or Egocentric, or even Hypocritic? - A Cross-Cultural Study of the Safety Priority Choice of Autonomous Car. TechRxiv. Preprint. https://doi.org/10.36227/techrxiv.16688941.v1

$\mathrm{DOI}$ 


\section{Altruistic or Egocentric, or even Hypocritic? \\ A Cross-Cultural Study of the Safety Priority Choice of Autonomous Car}

Ono, Shigeharu, ${ }^{*}$ Okazaki, Yumi, $†$ Kanetsuna, Keigo, $¥$ Mizumoto, Masaharu§

How should the autonomous car behave when faced with an unavoidable accident? In particular, in situations where either the driver or the pedestrian will inevitably be killed, whose safety should the autonomous car prioritize? There can be cases in which sacrificing the driver would be the best solution to maximize lives and minimize deaths. And we also need to consider the possibility that the victim could be ourselves. In this cross-cultural study with participants from US, Japan, and China, we investigated the drivers' safety setting preferences and found some interesting cultural differences in such preferences.

\section{Introduction}

When driving a car, we need to continuously check our surroundings for safety and perform precise maneuvers in response to the road conditions. This means that we are processing multiple tasks simultaneously while we drive. As a result, carelessness can easily occur, and this is a hotbed of traffic accidents. Previous researches show that human factors cause most traffic accidents [1].

In recent years, autonomous cars that can drive without human operations have been developed for practical use. The widespread use of autonomous cars is expected to lead to a significant reduction in traffic accidents [2][3]. However, on actual roads, multiple factors of traffic conditions such as weather, road surface condition, as well as speed, pedestrians, other cars, etc. change in complex ways. It is extremely difficult for autonomous cars to drive stably at all times while being affected by all these factors [4][5]. Therefore, even if autonomous cars reach the stage of practical use, traffic accidents involving autonomous cars are still expected to occur.

So, how should the autonomous car behave when faced with an unavoidable accident? In particular, in situations where either the driver or the pedestrian will inevitably be killed, whose

\footnotetext{
* Japan Advanced Institute of Science and Technology

${ }^{\dagger}$ Japan Advanced Institute of Science and Technology

${ }^{\ddagger}$ Japan Advanced Institute of Science and Technology

$\S$ Japan Advanced Institute of Science and Technology
} 
safety should the autonomous car prioritize? As autonomous cars become more widespread, this kind of moral dilemma is attracting more and more attention.

"Trolley problem" is a classic example of moral dilemma. In the standard trolley problem, participants have to choose between sacrificing five people fixed in another path by doing nothing against the running trolley or sacrificing one person to save five people in the path by moving a lever [6][7]. Previous researches applied the trolley problem to the context of autonomous cars. For example, Awad et al. [8] conducted a large-scale survey to compare the selection tendency of moral dilemma questions in various situations across cultures. The results suggested that there are cultural differences in selection tendencies. More specifically, multiple cultural backgrounds, such as religious beliefs and history, influence the selection.

However, even though their study involved the choice in which the driver becomes the victim to save the pedestrians, the participants did not judge the cases particularly from the driver's perspective. As mentioned by Lin [9] and Nyholm \& Smids [10], it is important to program the $\mathrm{AI}$ of autonomous car to minimize harm. However, there can be cases in which sacrificing the driver would be the best solution to maximize lives and minimize deaths [9]. And we also need to consider the possibility that the victim could be ourselves [11]. As more people will purchase and drive autonomous cars in the future, it is imperative to investigate people's actual preference of the safety priority settings when they themselves are to drive.

The driver's perspective is important especially when studying people's choice in moral dilemma. From the third-person perspective, choosing the option that prioritizes the children's or pregnant pedestrians' safety over the drivers' may be reasonable. But can we make the same decision when we are driving an autonomous car? Would you be willing to buy and drive a car that can kill you if the situation allows? For example, even if we and others choose the same action, our moral judgments of our own action and other people's action may not be the same [11]. This kind of asymmetry is all the more expected in moral dilemma.

The present study therefore explores people's choice in the face of a possible moral dilemma situation from the driver's perspective, through asking which safety setting of the car they prefer, the pedestrian-first or the driver-first, and its possible cultural variance. The study of Bonnefon, Shari, \& Rahwan [13] has already shown that while people agree that autonomous cars make utilitarian decisions, they prefer automated vehicles that protect themselves. Let us call this egocentric asymmetry. One of the two central aims of our study is to examine another dimension of the egoistic bias: hypocrisy. Basically, there are at least three kinds of autonomous cars in relation to the safety prioritization: (a) cars that prioritizes pedestrians' safety, (b) cars that prioritizes the driver's safety, (c) cars that the driver can choose which to prioritize (personal 
ethics setting) [11]. The important difference of (c) with the first two is that the driver can secretly set (and change) the safety priority. If both type-(a) cars and type-(b) cars are on the market and their priority settings are widely known, then, one's choice of the safety setting is publicly known and it is expected that there will be a tendency to choose (a)-type car if people consider the impression it gives to other people and (b)-type car if they do not. This tendency can be shown through comparing the rate of publicly choosing (b)-type car with the rate of privately choosing the driver-first setting for (c)-type car. If the former rate (in the public choice condition) is much lower than the latter rate (in the private choice condition), then we call such tendency hypocrite bias here.

It is natural to expect cultural variance in this tendency. Awad et al. [14] explained the cultural differences of judgments in their moral machine experiment using the notion of relational mobility. Relational mobility refers to the number of opportunities that people have to establish new relationships [15]. In a society with low relational mobility, people tend to avoid making decisions that do not make a good impression on society [14]. On the other hand, in a society with high relational mobility, the percentage of people making decisions that do not make a good impression on society increases [14]. In general, it is assumed that East Asian societies, including Japan and China, are those with low relational mobility, and North American society is one with high relational mobility [16].

Since driver's prioritizing the pedestrians' safety looks altruistic and prioritizing the driver's own safety looks egocentric, and, in general, altruistic choices give a better impression to other people than egocentric choices, we expect that, in a society with low relational mobility, more people choose the pedestrian-first setting in the public choice condition than in the private choice condition, and therefore the hypocrite bias is likely to be observed in such a society, or at least expected to be stronger there than in a society with high relational mobility.

Another aim of our study is to investigate the possible cultural variance of the safety priority preference itself, between the pedestrian-first setting and the driver-first setting of autonomous car from the driver's perspective. The results of "Moral Machine" study by Awad et al. [8] showed that Japanese people especially tended to prioritize pedestrians' safety over drivers compared to people in other regions. However, there the participants were not judging the situations particularly from the driver's perspective. We therefore examine whether the same pattern can be observed even when they choose the setting from the driver's perspective.

As mentioned, driver's prioritizing the pedestrians' safety looks altruistic. However, even if one chooses the pedestrian-first setting, the reason may not be so altruistic. It might be that one simply does not want to take responsibility in an accident involving pedestrians, assuming that 
one would be blamed for not choosing the pedestrian-first setting in such a case. Similarly, even if one chooses the driver-first setting, the reason may not be so egoistic. One might be simply optimistic about the AI technology of autonomous car, thinking that even under the driver-first setting, the autonomous car should be able to avoid accidents better than human drivers, so that the driver does not need to consider one's own responsibility. Thus, the underlying assumption about the responsibility, or who is responsibility for avoiding the accident, where the safety is left either to the driver's ethics or to the AI technology, may explain the people's choice.

In order to distinguish these views, we added a question about responsibility attribution, asking which side (the driver or the AI) is to be responsible for the fatal accident which would have been avoided if the driver had chosen the pedestrian-first setting but actually chose the driver-first setting. Those who chose the pedestrian-first setting simply to avoid the responsibility would blame the driver if they had chosen the driver-first setting. Those who chose the driver-first setting simply because they are optimistic about AI assume that the $\mathrm{AI}$ (or the manufacturer) is responsible for whatever accident involving an autonomous car, so that all the drivers should better choose the driver-first setting anyway.

Note that, although, assuming the existence of driver, we do not consider fully driverless-car (level 5 of autonomous driving levels of The US National Highway Traffic Safety Administration (NHTSA) 2013), the setting of pedestrian-first or driver-first is relevant from cars in which the human driver is mainly in control (level 1-2) to fully automated cars that a human driver can cede full control to the car whenever one chooses (level 3-4). Since the level of the driver's control affects the strength of blame on the driver's selfish choice [17], in our study we fixed the level of the driver's control ("Driver-Programmed condition" in [17]) throughout the groups and conditions.

Alternatively, there may be deeper reason for the safety priority choice, that is, one's preference for a particular balance or relation between the self and the society, which can be understood as a kind of what is called social value orientation (SVO) [18][19] (though this context is not directly about cooperation and competition). Thus, those who choose the pedestrian-first setting are certainly pro-social, but may not be particularly altruistic. They may be just (horizontal) collectivists, assuming that everyone must do the same. Similarly, those who choose the driverfirst setting are pro-self, but may not be particularly egocentric, like those who think that the pedestrian-first setting is actually ideal, but when it comes to one's own car, prefers the driverfirst setting. Rather, they may be just (horizontal) individualists, assuming that everyone should protect oneself by one's own way. 
In order to distinguish these underlying attitudes, we asked another question, about whether the car company should produce the cars that prioritizes pedestrians' safety or the cars that prioritizes the driver's safety.

As mentioned above, according to the results of Awad et al. [8], Japanese people tend to prioritize pedestrians' safety over drivers. If this is also the case for the Japanese even when they choose the priority from the driver's perspective, that is most likely to be because they are collectivists, which, in turn, can be explained by Japanese society's low relational mobility. On the other hand, people in a high relational mobility society are expected to freely choose the driver-first setting, even recommending others to do the same, and if so, they are rather individualists.

Thus, these additional questions not only supplement the first question of the preference of the safety setting of the autonomous car by distinguishing the reasons for participants' choice, but also help predict the cultural difference of the preference of the safety setting.

This study therefore would provide the data about people's views on the ethical issues raised by Nyholm \& Smids [10] from the driver's perspective, which is important for what type of autonomous car should be produced. Since the results would reflect the drivers' (possible customers') attitudes, which might vary from culture to culture, they are all the more for it important for both car companies and law-makers. In this study we use participants from Japan, China, and the United States for cultural comparison of people's selection tendency.

\section{Method}

Participants were recruited through crowdsourcing sites (Japan: Yahoo! Crowdsourcing, China: Baidu Cloud's questionnaire survey function, USA: Amazon M-Turk). Participants were randomly divided into two groups, $\mathrm{A}$ and $\mathrm{B}$, and were asked to answer questions. The first question (Q1) is to choose between the pedestrian-first setting and the driver-first setting of an autonomous car they are to drive. Group A participants choose the safety priority of the (c)-type car (the private choice condition). Group B participants choose between the (a)-type car and the (b)-type car (the public choice condition). (See Appendix for the material used.)

The second question (Q2) is to probe the difference of the assumptions of participants about the responsibility in the case of an unavoidable accident involving an autonomous car with a victim. There, an autonomous car that prioritizes the driver's safety causes an accident and kills a pedestrian, and the question is who is responsible, the driver or the AI (or its design manager). Corresponding to the type of the car mentioned in Q1, there were two versions of the vignette for 
Q2 for group A and group B (though this difference did not make any difference to the answers to Q2).

The third question (Q3) was about the ideal car production, asking both groups whether car companies should produce the (a)-type cars or the (b)-type cars. Since this is not a question particularly from the driver's perspective, it asks one's ideal car-type to be on the road. So, if there was any gap between answers to this question and the answers to Q1, that constitutes what we called the egocentric asymmetry. But the point of this question was to identify, in combination with Q1, participants' social value orientation. Thus, among those who chose the pedestrian-first setting in Q1, only those who chose the (a)-type car (with pedestrian-first setting) are truly altruistic (call them idealistic altruists), and those who chose the (b)-type car (with driver-first setting) are just individualists (or principled individualists). Among those who chose the diverfist setting in Q1, only those who chose the (a)-type car are egocentric (call them realistic egocentrics), and those who chose the (b)-type car are rather individualists (or principled individualists).

Finally, as demographic questions, we asked, in addition to gender and age, a question about how frequently participants drive a car (either more than once a week or not), considering that the participants' driving frequency might affect the self-identification of themselves as a driver, with more driving experiences encouraging to take the driver's perspective more seriously.

We compared the selection tendencies of peoples in Japan, China, and the United States. In the U.S. survey, we also asked the native language of the participants, and excluded participants whose native language was not English or was English outside the U.S. (e.g., India), in order to secure the cultural comparison. The number of participants analyzed was 205 in Japan (Male: 153, Female: 49, Non-binary: 3, Mean age: 45.0, SD: 9.54), 228 in China (Male: 129, Female: 99, Mean age: 32.5, SD: 7.9), and 250 in USA (Male: 128, Female: 121, Non-binary: 1, Mean age: 35.9, SD: 11.7).

Since it is generally thought that Japanese and Chinese societies (or Eastern societies in general) are low relational mobility societies while the American society (or Western societies in general) is one with high relational mobility, we expect that we will observe stronger hypocrite biases in Japanese and Chinese participants than in Americans. And if the Japanese and Chinese are truly hypocritic, there will be no cultural difference in the private setting condition.

Also, as for the priority setting in general, we expect that both Japanese and Chinese tend to choose the pedestrian-first setting with no significant cultural difference, and Americans tend to choose the driver-first setting. If this is due to the difference of the assumption about the responsibility, most Japanese and Chinese would attribute responsibility to the driver, while 
most Americans would to the AI, in the second question. If it is more to participants' SVO, that is because Japanese and Chinese are more principled collectivists than idealistic altruists, and Americans are more principled individualists than realistic egocentrics.

\section{Results}

\subsection{Model Estimation}

We first conducted logistic regression analyses to see if age, gender, and driving frequency predict the safety priority choice (answers to Q1) for each country, and found that any of these factors did not significantly predict the choice in all cultures.

We then constructed logistic regression models with the safety priority choice as the dependent variable, and group (G), responsibility attribution (R), and ideal car-type (I) as predictor variables. Equation (1) is the base model, and equation (2) is a model with interactions (the interaction model).

$\operatorname{logit}(\Pi)=\alpha+B_{1} D_{G}+B_{2} D_{R}+b_{3} D_{I}$

$\operatorname{logit}(\Pi)=\alpha+B_{1} D_{G}+b_{2} D_{R}+B_{3} D_{I}+B_{4} D_{G} * D_{R}+b_{5} D_{G} * D_{I}+b_{6} D_{R} * D_{I}$

Likelihood ratio tests showed that (1) was a significantly better fit than the null model in all countries. Also, (2) was not a significantly better fit model than (1) in all countries. The Wald tests of the three factors in (1) showed that, in all countries, the ideal car-type choice was a significant predictor of the safety priority choice, but while in the Chinese data all three factors (group, responsibility, ideal car-type) were significant predictors of the priority choice, in the Japanese and American data, other two factors were not. None of the interaction terms in (2) was a significant predictor.

Finally, we conducted a Wald test on the model in which the element of culture (C) was added as an independent variable to the base model, comparing the three countries pairwise, shown below as equation (3).

$$
\operatorname{logit}(\Pi)=\alpha+\beta_{1} D_{C}+b_{2} D_{G}+b_{3} D_{R}+B_{4} D_{I}
$$

Against our prediction, the regression coefficients of culture significantly predicted the results only in Japan-China and Japan-US comparative models, but not in the China-US model (Table $1)$. 


\begin{tabular}{|c|c|c|c|}
\hline & $\mathrm{JP}^{*} \mathrm{CN}$ & $\mathrm{JP}^{*} \mathrm{US}$ & $\mathrm{US}^{*} \mathrm{CN}$ \\
\hline Intercept & 6.672 & 19.730 & 30.587 \\
\hline (p-value) & $(0.0098)$ & $(<0.001)$ & $(<0.001)$ \\
\hline Culture & 8.708 & 6.417 & 0.439 \\
\hline (p-value) & $(0.0032)$ & $(0.011)$ & $(0.507)$ \\
\hline Group & 13.309 & 4.162 & 8.880 \\
\hline (p-value) & $(<0.001)$ & $(0.041)$ & $(0.0029)$ \\
\hline Responsibility (Q2) & 7.322 & 1.915 & 4.810 \\
\hline (p-value) & $(0.0068)$ & $(0.166)$ & \\
\hline Ideal Car-Type (Q3) & 120.132 & 131.756 & 130.197 \\
\hline (p-value) & $(<0.001)$ & $(<0.001)$ & $(<0.001)$ \\
\hline
\end{tabular}

Table 1: Wald statistics of culture model

We then compared each of three instances of (3) with the corresponding interaction model that uses the cultural factor as a focal independent variable, shown below as equation (4).

$$
\operatorname{logit}(\Pi)=\alpha+B_{1} D_{C}+b_{2} D_{G}+b_{3} D_{R}+b_{4} D_{I}+b_{5} D_{G} * D_{C}+b_{6} D_{R} * D_{C}+b_{7} D_{I} * D_{C}(4)
$$

According to likelihood ratio tests, (4) was a significantly better fit than (3) only in the JapanChinese comparison ( $p$ 0.0015). However, according to the Wald tests of (4), the overall cultural difference disappeared there (culture failed to be a significant predictor in any instance of (4)) and only the interaction term of culture and ideal car-type (answers to Q3) was a significant predictor in the Japanese-Chinese comparison and the Japanese-American comparison. No other interaction term was a significant predictor (Table 2). 


\begin{tabular}{|c|c|c|c|}
\hline & $\mathrm{JP}^{*} \mathrm{CN}$ & $\mathrm{JP}{ }^{*} \mathrm{US}$ & $\mathrm{US}^{*} \mathrm{CN}$ \\
\hline Intercept & 10.563 & 10.563 & 8.692 \\
$(\mathrm{p}$-value) & $(0.0012)$ & $(0.0012)$ & $(0.0032)$ \\
\hline Culture & 3.002 & 0.318 & 3.395 \\
\hline (p-value) & $(0.083)$ & $(0.572)$ & $(0.065)$ \\
\hline Group & 3.426 & 3.426 & 10.635 \\
(p-value) & $(0.064)$ & $(0.064)$ & $(0.0011)$ \\
\hline Responsibility (Q2) & 0.88 & 0.88 & 4.643 \\
(p-value) & $(0.348)$ & $(0.348)$ & $(0.031)$ \\
\hline Ideal Car-Type (Q3) & 46.938 & 46.938 & 52.884 \\
$(\mathrm{p}$-value) & $(<0.0001)$ & $(<0.0001)$ & $(<0.0001)$ \\
\hline Culture*Group & 0.044 & 0.89 & 2.257 \\
\hline (p-value) & $(0.834)$ & $(0.345)$ & $(0.133)$ \\
\hline Culture*Responsibility & 0.15 & 0.069 & 0.711 \\
\hline (p-value) & $(0.698)$ & $(0.792)$ & $(0.399)$ \\
\hline Culture*Ideal Car-Type & 10.285 & 4.972 & 2.254 \\
(p-value) & $(0.0013)$ & $(0.026)$ & $(0.133)$ \\
\hline
\end{tabular}

Table 2: Wald statistics of culture interaction model

\subsection{Comparisons of Individual Results}

Let us also look at the detail of the results of questions individually based on the results of these analyses. Here we use Fisher's exact test (two-tailed) for statistical significance and phi for effect size (where effect size is small if $\phi=0.1$; medium if $\phi=0.3$; large if $\phi=0.5$ ).

\section{Answers to Q1:}

Figure 1 is the percentage of participants who selected the driver-first setting. (The bars in the graphs are all $95 \%$ confidence intervals throughout this section.) The rate of participants who chose the driver-first setting in group A (private choice condition) was significantly lower than in group B (public choice condition) in Japanese and Chinese surveys, which are the opposite effects of the hypocrite bias. 


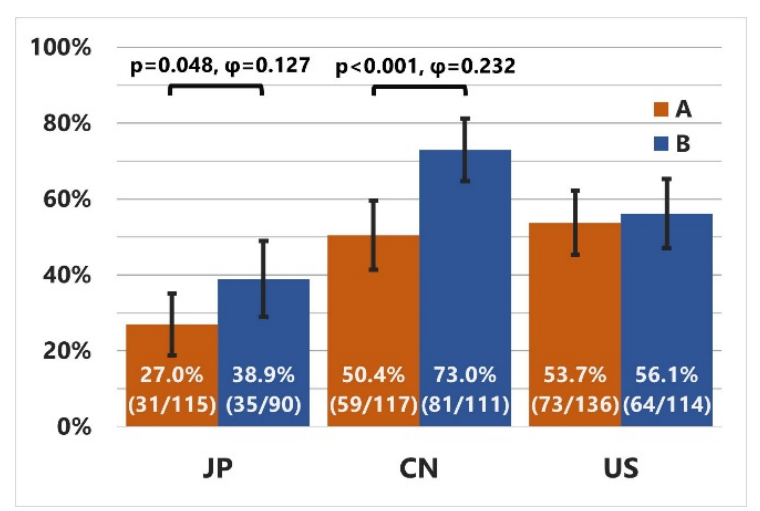

Figure 1: Rate of participants who select the driver-first setting in Q1

The rate of the Japanese driver-first choice was consistently significantly lower than those of Chinese and Americans both in group A (compared with Chinese: $p=0.0003, \varphi=0.24$, Americans: $\mathrm{p}<0.0001, \varphi=0.27$ ) and in group B (compared with Chinese: $\mathrm{p}<0.0001, \varphi=0.34$, Americans: $\mathrm{p}=0.017, \varphi=0.17$.

\section{Answers to Q2:}

Since there was no significant difference in answers to Q2 between group A and group B, we only report the compound data here. Figure 2 is the percentage of respondents who selected the AI (or design manager) as responsible for the accident. The rates of Chinese and American participants were significantly higher than that of Japanese participants.

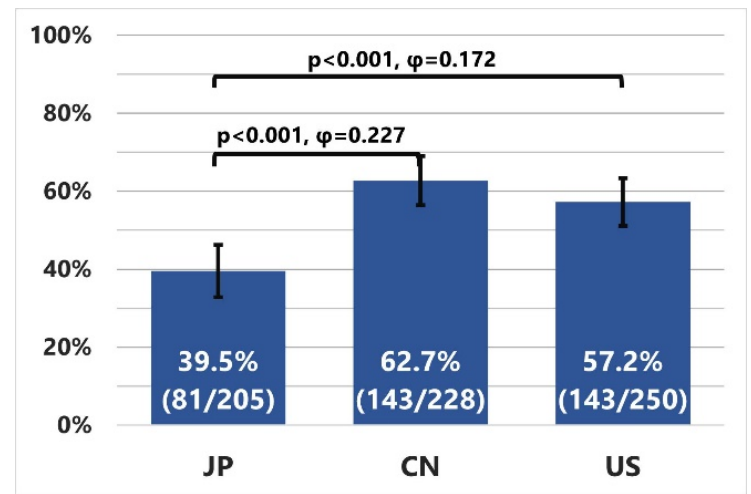


Figure 2: Rate of participants that select the AI in Q2

Figure 3 shows the rates of participants who selected the AI in Q2 broken down by the answers to Q1. In all countries, those who selected the driver-first setting in Q1 had a significantly higher rate of selecting the AI than the rate of those who selected the pedestrian-first setting. But the difference is much larger (in terms of effect size) in the Japanese results. Also, even though there was no significant cultural difference among those who chose the driver-first setting, among those who chose the pedestrian-first setting, the Japanese participants who attributed responsibility to the $\mathrm{AI}$ was significantly lower than both Chinese $(\mathrm{p}=0.011, \varphi=$ 0.18 ) and Americans $(p=0.025, \varphi=0.14)$, suggesting the Japanese who preferred the pedestrian-first setting as a driver tend to blame the driver much more than Chinese and Americans.

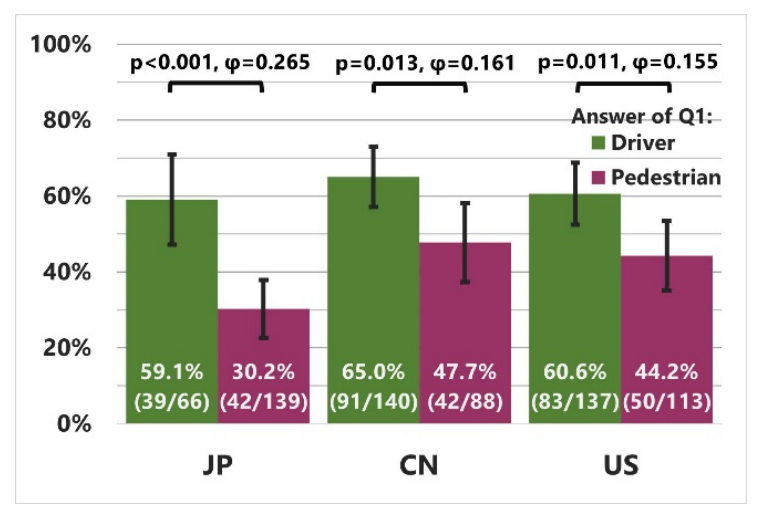

Figure 3: Rate of participants who select the $\mathrm{AI}$ in $\mathrm{Q} 2$ in relation to the answers to $\mathrm{Q} 1$

\section{Answers to Q3:}

Figure 4 shows the rates of participants who chose the car with the driver-first setting in response to Q3. (One American subject did not answer this question.) To examine the egocentric asymmetry, we compared this result with answers to Q1, and found that the rates of the Chinese participants and the American participants who chose the driver-first setting were significantly lower (despite the small effect sizes) than their answers to Q1 (Chinese: $p=0.018, \varphi=0.11$; Americans: $p=$ $0.020, \varphi=0.11)$. 


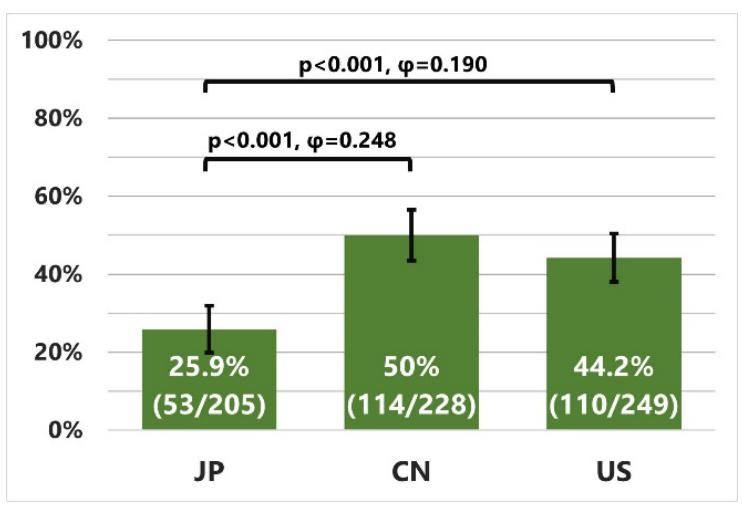

Figure 4: Rate of participants who chose a driver-first setting in Q3

However, the breakdown of the answers by the answers to Q1 shows that participants were surprisingly consistent between these questions, and there was no cultural difference in the rate of those who chose the driver-fist setting (figure 5).

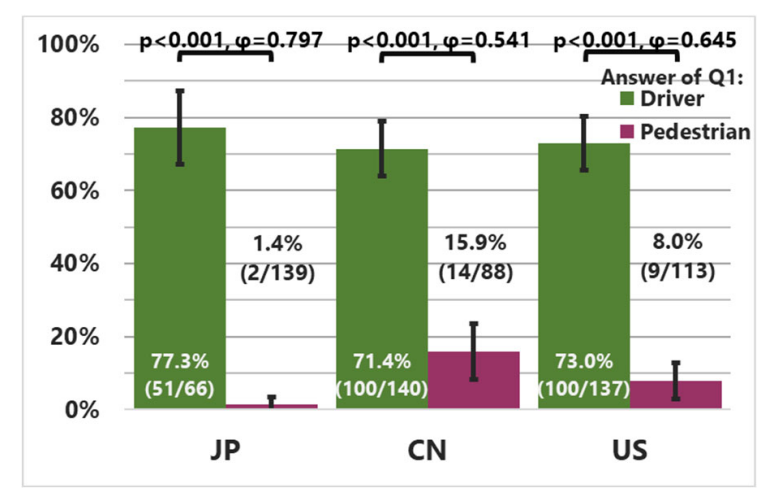

Figure 5: Rate of participants who chose a driver-first setting in Q3 in relation to Q1

Still, the cultural difference of the overall distribution of social value orientation was still impressive (table 2). Nearly $70 \%$ of Japanese were collectivists, while only $30 \%$ of Chinese were. The distributions of Chinese and Americans were generally very similar, with striking contrasts with the Japanese distribution. 


\begin{tabular}{|l|c|c|c|}
\hline \multicolumn{1}{|c|}{ JP } & CN & US \\
\hline $\begin{array}{l}\text { principled } \\
\text { collectivist }\end{array}$ & $67 \%$ & $32 \%$ & $41 \%$ \\
\hline $\begin{array}{l}\text { idealistic } \\
\text { altruist }\end{array}$ & $1 \%$ & $6 \%$ & $4 \%$ \\
\hline $\begin{array}{l}\text { realistic } \\
\text { egoist }\end{array}$ & $7 \%$ & $18 \%$ & $14 \%$ \\
\hline $\begin{array}{l}\text { principled } \\
\text { individualist }\end{array}$ & $25 \%$ & $44 \%$ & $41 \%$ \\
\hline
\end{tabular}

Table 3: Distribution of Social Value Orientation

As the figure 6 shows, if we compare the rates of principled collectivists and the rates of principled individualists, the rate of Japanese collectivists is significantly higher than those of Chinese and Americans, and the rate of Japanese individualists are significantly lower than the other two.

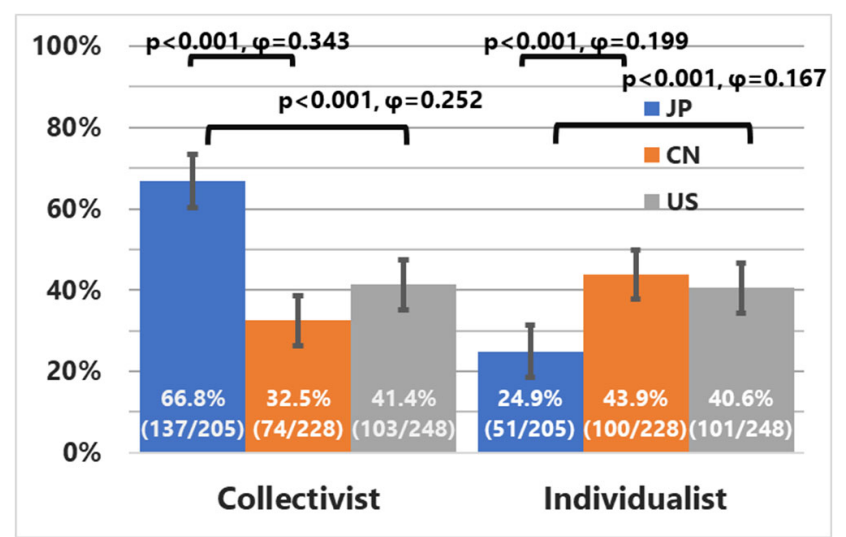

Figure 6: Rates of Collectivists and Individualists in Three Countries

\section{Discussion}

The results of our study were not consistent with both of the two main hypotheses. As for the first hypothesis (about hypocrite bias), regardless of the relational mobility, the hypocrite bias was not found in any of the cultures. Indeed, the tendency to choose the driver-first choice was significantly higher in the public choice condition (group B) than in the private choice condition (group A) in the Japanese and Chinese results (figure 1), but this is the opposite effect of the hypocrite bias. One possible account of this is that, compared to the private ethics setting, choosing a car to purchase is not taken as particularly ethical decision for Japanese and Chinese, where the ethical decision is already done by the car company, and therefore the psychological bar (to choose the driver-first setting) is lowered there, though this is only a 
psychological effect, not a principled view, since there was no difference in responsibility attribution between A and B in the answers to Q2 either of Japanese or Chinese.

As for the second hypothesis (about cultural variance of the safety priority choice), there was no difference between Chinese and Americans, while the difference between Japanese and Chinese was significant. In particular, Japanese participants showed significantly lower rates of the driver-first setting than Chinese and American participants in the answers to Q1 in both group A and group B (figure 2). The prediction based on relational mobility was consistent with the low Japanese rates and the high American rates of the driver-first setting, but the high rates of the driver-first setting in Chinese participants were contrary to our expectation. Indeed, in group B (the public setting condition), the rate of the driver-first setting of Chinese was even significantly higher than that of Americans $(p=0.0002, \varphi=0.18)$. These results suggest that the cultural variance of the safety priority choice cannot be explained by relational mobility alone.

Rather, logistic regression analyses showed that only the ideal car-type (Q3) was a significant predictor of the safety preference in all countries, and only the interaction term of the culture and the ideal car-type was a significant predictor in cultural comparisons. These suggest that SVO is the main predictor of the safety priority in both the intra-cultural and the cross-cultural contexts. (while the cultural difference of SVO itself cannot be explained by relational mobility).

Also, even though we found the egocentric asymmetry in Chinese and Americans, even if this was a within-subjection comparison, the strong consistency of participants between answers to Q1 and Q3 suggests that, there being only few egocentrics and altruists, the cultural difference is mainly due to the difference of the collectivist/individualist tendencies.

\section{Conclusion}

We expected that low relational mobility of the Japanese and Chinese societies would generate what we called hypocrite bias, and the relational mobility in general would explain the cultural variance of the safety priority choice. We conducted surveys with Japanese, Chinese, and American participants to test such hypotheses, but we obtained rather contrary effects of hypocrite bias in the Japanese and Chinese results, and the contrary tendency of safety priority choice in Chinese.

However, we believe that the results provide meaningful data that cannot be obtained from the standard moral dilemma surveys, which selects victims of accidents from a thirdperson's perspective. In particular, we found the consistently higher rates of the Japanese preference of the pedestrian-first setting, and this strong tendency in Japanese even from the driver's perspective supplements the results of Awad et al [8]. Moreover, the similarity of the 
higher rates of the driver-first setting of Chinese and Americans despite the difference of the relational mobility, is already interesting enough. In this connection, we should also note the participants' consistency between answers of Q1 and Q3, which suggests the polarity of the collectivist and the individualist tendencies, where, unlike the ordinary East/West or low/high relational mobility distinction, the contemporary Chinese society rather belongs to the individualist side at least concerning the ethics of autonomous car. This unexpected dissociation between relational mobility and SVO found in Chinese deserves an independent further exploration.

Finally, if the psychological bar account of the opposite effect of the hypocrite bias discussed in the last section is correct (which is left for a future study), that is an important fact for both car companies and law makers, and even support the claim of those who think that the mandatory ethics setting is better for the society, so that only the pedestrian-first cars should be on the market [11].

\section{Appendix: The materials used for the American survey}

Q1

\section{Group A}

A1

You bought a car that has an auto driving AI.

Normally you drive the car by yourself, but it has an auto braking and an auto steering to avoid collisions. In addition, you need to choose in advance which safety is to be prioritized, the driver or pedestrians, in the case of an emergency such as a collision that cannot be avoided by the auto braking or the auto steering.

Your setting cannot be seen by other people.

Q1 Which setting do you choose?

1) Setting that prioritizes driver's safety

2) Setting that prioritizes pedestrian's safety

\section{Group B}

B.1

You are going to buy a car that has an auto driving AI. 
Normally you drive such cars by yourself, but they have an auto braking and an auto steering to avoid collisions. In addition, the cars have an AI that is preinstalled to prioritize either the safety of the driver or the safety of pedestrians in the case of an emergency such as collisions that cannot be avoided by the auto braking or the auto steering.

Which AI is preinstalled depends on the type of the car, and which type of car has which $\mathrm{AI}$ is widely known.

Now, you are having trouble deciding which one to buy, a type-A car or a type-B car. The type-A car and the type-B car look very different and it is well-known that the type-A car has an $\mathrm{AI}$ that prioritizes the driver's safety and the type-B car has an $\mathrm{AI}$ that prioritizes pedestrians.

If you buy a type-A car, your safety as a driver is prioritized, but there are many people who accuse the drivers of this type of car as selfish. On the other hand, if you buy a type-B car, the safety of pedestrians is prioritized, and therefore there are few people who accuse the drivers of such a car, but some people describe them as hypocrites.

1) Type-A car

2) Type-B car

Q2

Group A

A.2 (Words in the square brackets were those of B2 for group B)

A car that has an auto driving AI had an accident. The victim of the accident was a 10-year-old elementary school boy. The boy was taking his dog for a walk, and then a ball flew from a nearby park. The dog chased the ball and strongly pulled the lead, taking the boy to the road, where the boy was hit by the car and died.

The car driver claimed that the auto driving AI was responsible for the accident. At the time of the accident, the driver was driving alone, observing the legal speed limit. The accident site was a single lane road on each side. There were sidewalks lined with roadside trees on both sides of the road and there were many oncoming cars.

It was not possible to avoid the accident by his braking because the boy emerged too suddenly from behind the roadside trees. There was therefore only a choice between mitigating collision with the boy, and avoiding the collision with the boy by swerving the car and thereby colliding with oncoming vehicles or the roadside trees.

At the time of the accident the car had been set to prioritize the driver's safety in the case of emergency [At the time of the accident, the driver was driving a type-A car]. Therefore, 
the auto steering that would put the driver in danger was not activated and only the auto braking system started working, which was not enough to avoid hitting the boy and save his life.

If the driver's car had not been a car with an auto driving AI, the driver might have swerved the car, which would have put him at risk of injury but would not have killed him, and the boy would not have been killed either.

The victim's family claimed that the driver was responsible for the accident. For, if the driver's car had been set to prioritize pedestrians' safety in the case of an emergency [For, if the driver's car had been a type-B car], the boy would not have been killed while the driver would have been put at the risk of injury but not of his life.

1) Auto driving $\mathrm{AI}$ (or design manager of $\mathrm{AI}$ )

2) Driver

\section{References}

[1] Mabrouk Touahmia. Identification of risk factors influencing road traffic accidents. Engineering, Technology \& Applied Science Research, 8(1):2417-2421, 2018.

[2] Rasheed Hussain and Sherali Zeadally. Au-tonomous cars: Research results, issues, and future challenges. IEEE Communications Surveys \& Tutorials, 21(2):1275-1313, 2018.

[3] Ibrar Yaqoob, Latif U Khan, SM Ahsan Kazmi, Muhammad Imran, Nadra Guizani, and Choong Seon Hong. Autonomous driving cars in smart cities: Recent advances, requirements, and challenges. IEEE Network, 34(1):174-181, 2019.

[4] Shizhe Zang, Ming Ding, David Smith, Paul Tyler, Thierry Rakotoarivelo, and Mohamed Ali Kaafar. The impact of adverse weather condi-tions on autonomous vehicles: how rain, snow, fog, and hail a ect the performance of a self-driving car. IEEE vehicular technology magazine, 14(2):103-111, 2019. 
[5] Li Tang, Yunpeng Shi, Qing He, Adel W Sadek, and Chunming Qiao. Performance test of autonomous vehicle lidar sensors under di erent weather conditions. Transportation research record, 2674(1):319-329, 2020.

[6] Philippa Foot. The problem of abortion and the doctrine of double e ect. Oxford Review, 5:5$15,1967$.

[7] Judith Jarvis Thomson. Killing, letting die, and the trolley problem. The Monist, 59(2):204$217,1976$.

[8] Edmond Awad, Sohan Dsouza, Richard Kim, Jonathan Schulz, Joseph Henrich, Azim Shari , Jean-Fran, cois Bonnefon, and Iyad Rah-wan. The moral machine experiment. Nature, 563(7729):59-64, 2018.

[9] Patrick Lin. Why ethics matters for autonomous cars. In Autonomous driving, pages 69-85. Springer, Berlin, Heidelberg, 2016.

[10] Sven Nyholm and Jilles Smids. The ethics of accident-algorithms for self-driving cars: An ap-plied trolley problem? Ethical theory and moral practice, 19(5):1275-1289, 2016.

[11] Jan Gogoll and Julian F Muller. Autonomous cars: in favor of a mandatory ethics setting. Science and engineering ethics, 23(3):681-700, 2017.

[12] Piercarlo Valdesolo and David DeSteno. The duality of virtue: Deconstructing the moral hypocrite. Journal of Experimental Social Psychology, 44(5):1334-1338, 2008.

[13] Jean-Francois Bonnefon, Azim Shari, and lyad Rahwan. The social dilemma of autonomous vehicles. Science, 352(6293):1573-1576, 2016.

[14] Edmond Awad, Sohan Dsouza, Azim Shari, lyad Rahwan, and Jean-Francois Bonnefon. Universals and variations in moral decisions made in 42 countries by 70,000 participants. Proceedings of the National Academy of Sciences, 117(5):2332-2337, 2020.

[15] Masaki Yuki, Joanna Schug, Hiroki Horikawa, Kosuke Takemura, Kosuke Sato, Kunihiro Yokota, and Kengo Kamaya. Development of a scale to measure perceptions of relational mobility in society. CERSS Working Paper, 75, 2007. 
[16] Masaki Yuki and Joanna Schug. Relational mobility: A socioecological approach to personal relationships. Psychology, 95:352-368, 2008.

[17] Ryan M McManus and Abraham M Rutchick. Autonomous vehicles and the attribution of moral responsibility. Social psychological and personality science, 10(3):345-352, 2019.

[18] Van Lange, P. A. M. Beyond self-interest: a set of propositions relevant to interpersonal orientations. Eur. Rev. Soc. Psychol. 11, 297-331, 2011.

[19] Moon, Chanki, Giovanni A. Travaglino, and Ayse K. Uskul. "Social value orientation and endorsement of horizontal and vertical individualism and collectivism: An exploratory study comparing individuals from North America and South Korea." Frontiers in psychology 9: 2262, 2018. 